\title{
TESTING AND EVALUATION OF SUPERCONDUCTING CABLES FOR THE LHC
}

\author{
R. Thomas ${ }^{\#}$ A. Ghosh, D. McChesney, and A. Jain, BNL, Upton, NY
}

\section{Abstract}

As one of the activities of the US-LHC Accelerator Project, BNL is testing short samples of superconducting cables that will be used in the main LHC dipoles and quadrupoles. The purpose of these tests is to verify that the reels of superconducting cables as supplied by the vendors meet the required critical current specifications. The short-sample testing facility and the computer-assisted testing techniques for acquiring the data will be described. We also describe the data analysis, data storage, and data transmission methods.

\section{APPROACH}

Electrical tests of cable samples must be done during cable production so that cable manufacturing problems may be detected as early as possible. During the period that CERN is procuring superconductor cable for the LHC project, BNL is providing resources to verify the superconducting properties of the cables. The BNL short-sample test facility is used to measure the critical current, $I_{\mathrm{ct}}$, of short ( $\leq 1$ meter) samples of LHC dipole and quadrupole cables.

The number of tests that must be performed is set by the cable production schedule and reaches a peak rate of about 20 samples per week. The total number of tests that will be performed over the six-years of the collaborative effort will exceed 3000 .

A large quantity of information needs to be acquired, analyzed, and stored. Also, reports on individual runs, summary sample reports, and compatible raw data files must be regularly transferred to CERN. Because the data handling tasks themselves present the largest challenges, the database is the key organizing structure for all other operations.

This approach is possible as a result of recent advances in networked databases and distributed objectoriented software programming. These allow software programs located anywhere on a network (either a local intranet or the world-wide Internet) to interact with a database using the client/server model.

As samples are received, information about each sample is entered into a table (CableTrack) in the database. When an assembly of four samples is placed in a

\footnotetext{
"Work supported by the U. S. Department of Energy, Contract No. DE AC02-98CH10886.

${ }^{t E}$ Email: thomas 1@bnl.gov
}

sample holder for testing, information about the assembly is entered into the CableAssembly table.

When the actual testing takes place, the data acquisition program interacts directly with the same database to give the user a drop-down list of assemblies from which he selects the assembly being tested. From that selection alone, the data acquisition program can obtain information from the remote database regarding the physical characteristics of the samples being tested and their instrumentation wiring. Fig. 1 shows the data flow and database interactions.

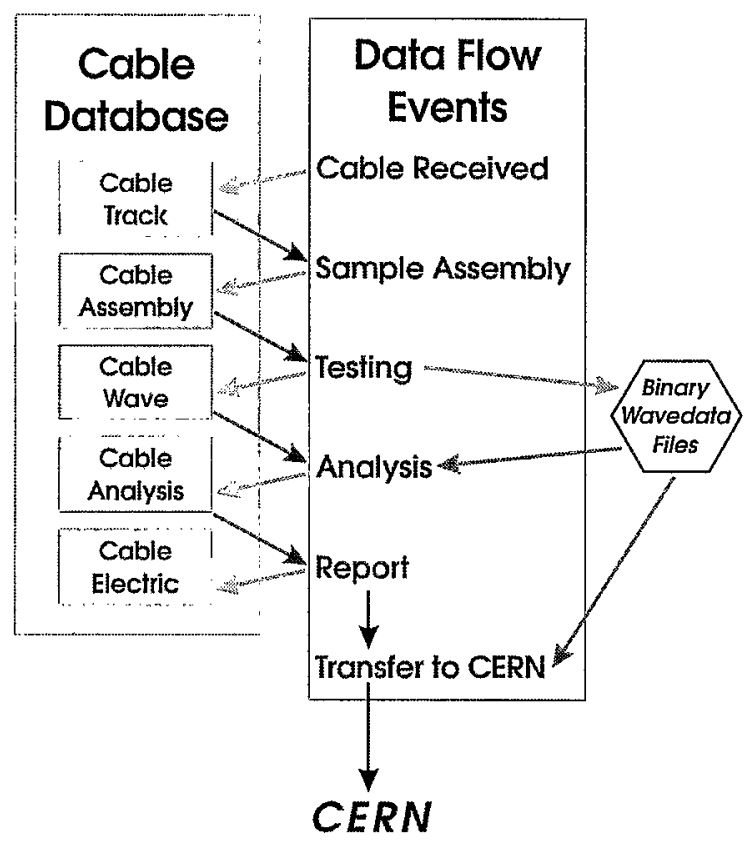

Figure 1. Selected tables of the Cable Database and interactions with the data flow events.

When data are acquired, values are written into the CableWave table of the database describing the experimental conditions and, the name of the raw binary data file (a copy of which is automatically placed on the network file server).

Once this information has been obtained, data analysis can be carried out on any machine connected to the network. (Software has been produced that loads data into workbooks, fits the data to a model, and writes results back into the CableAnalysis table of the database.) Other programs allow the user to select records from the CableAnalysis table which the program then uses to produce summary reports. 
Finally, result files, plain-text raw data files, summary reports are shipped to CERN using ftp (file transfer protocol). Although there is a program to assist in this step, it has not yet been fully automated. Also, the construction of a web page that allows those at CERN to follow the status of any sample is still under development.

\section{SHORT SAMPLE TEST FACILITY}

The standard tests are performed at $4.2 \mathrm{~K}$. Approximately $10 \%$ of the cable samples are being tested in superfluid helium at $1.9 \mathrm{~K}$ to determine the consistency of the $\Delta \mathrm{H}$ shift of the $\mathrm{I}_{\mathrm{ct}}$ vs. $\mathrm{H}$ curve from $4.2 \mathrm{~K}$ to $1.9 \mathrm{~K}$. Four samples can be tested in one cool-down of a single test station (cryostat), so two additional test stations are being built to meet the LHC production schedule.

The layout of the four samples in the sample holder is shown in Fig. 2. The sample holder is placed in a cryostat which also holds a superconducting dipole magnet which can produce fields as high as $-9 \mathrm{~T}$ at $1.9 \mathrm{~K}$ [1]. The magnet is energized by a $8.5 \mathrm{kA}$ power supply; a

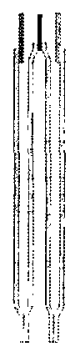
superconducting switch allows it to operate in the persistent mode.

As the critical current of the cable will generally be in the range of $10-20$ $\mathrm{kA}$, two $15 \mathrm{kA}$ supplies are operated in parallel to supply the cable test current.

Figure 2. Sample arrangement In addition to the in the sample holder. power supplies and their controls and safety devices (quench detectors and fault detection circuits), the facility includes the instrumentation necessary for controlling the cryogenic environment and for measuring the critical current of the samples under test.

Two computer systems are used: 1) a cryogenic system that monitors liquid helium levels, temperatures, and pressures and both sets and monitors the flow of helium through the gas-cooled leads, and 2) an experimental system for controlling the sample power supply, taking data, and storing data in the SQL database.

\section{TEST}

The goal of the short-sample cable tests is to determine the transport critical current of the samples. This critical current is a function of temperature and the magnetic field. In order to obtain standardized results for a specified reference temperature and reference field, raw data must be obtained for several applied fields with the samples at the temperature of the helium bath; that is, one acquires a set of critical currents, $I_{\mathrm{c}}$, at bath temperatures, $T_{\mathrm{b}}$, and applied fields, $B_{\mathrm{a}}$, for each sample.

By pairing samples which are believed to have similar properties, it is possible to acquire data on two samples simultaneously. Also, as there are three gascooled leads connecting the two sample pairs to the sample power supplies (see Fig. 2), four samples can be tested in a single cool-down, with the pairs being tested sequentially.

To acquire the $I_{\mathrm{c}}$ data, the operator first sets the current in the dipole magnet for the desired applied field, then connects the sample power supply to the first sample pair to be tested. He then enters the required information into the data acquisition program regarding the ramp rate and maximum current for the ramp on the form shown in Fig. 3.

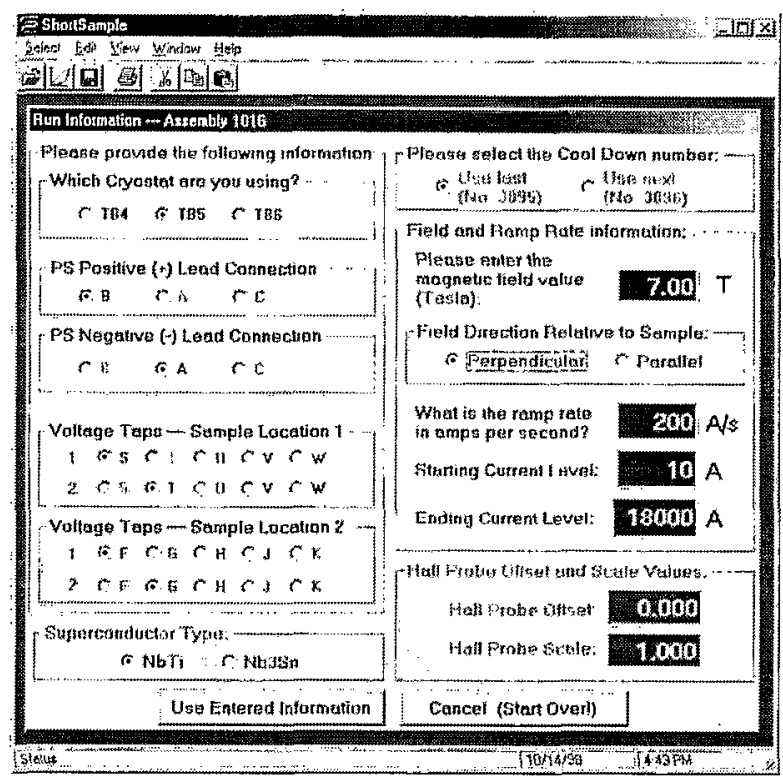

Figure 3. User form for entering run information.

Three high-resolution volt meters are triggered simultaneously as the current is ramped to obtain the voltage drops across the two samples and the current level as a function of time, as shown in Fig. 4.

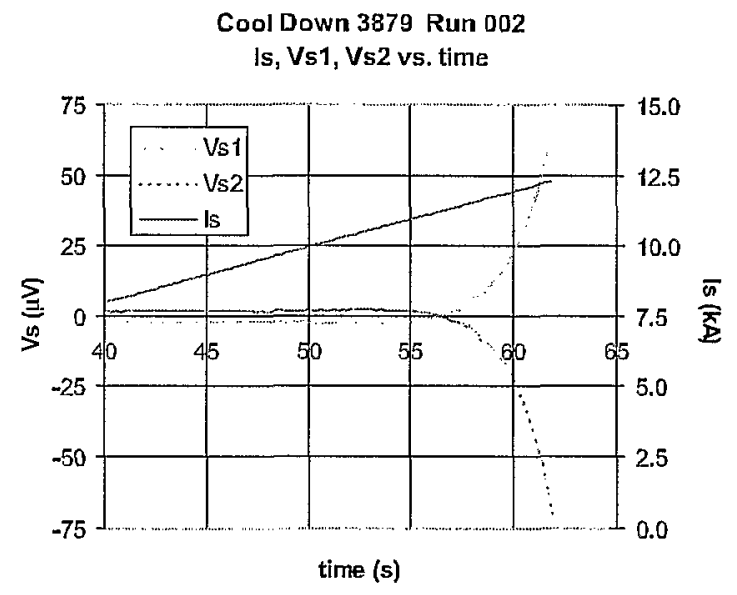

Figure 4. Actual signals for low-noise conditions. 
Several sets of data are obtained at different dipole field levels for each sample pair.

\section{ANALYSIS AND REPORTING}

Once the raw data have been collected, analysis can begin on any workstation connected to the network, since all the required information for each run can be extracted from the database. On many occasions there is considerable electrical noise visible on the signals, primarily as a result of phase and voltage flicker that is produced by the synchrotron accelerators located nearby. When this is the case, the use of a model and a fitting procedure allow the value of $I_{\mathrm{c}}$ to be extracted. (See Fig. 5.)

\section{Cool Down 3888 Run 017 01098001A-1 AB Vs vs. Is}

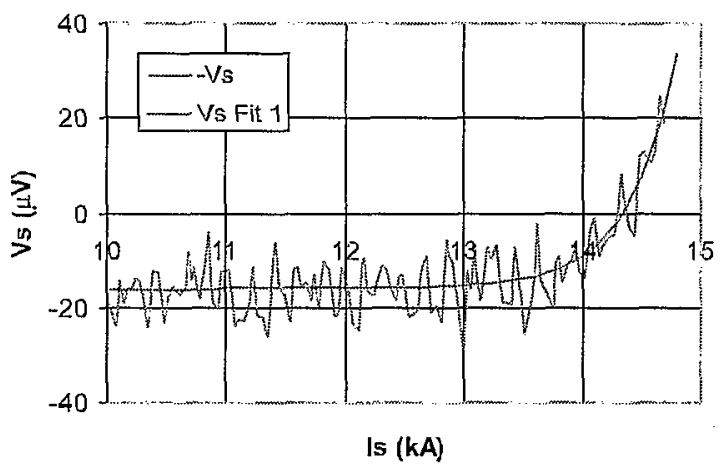

Figure 5. Noisy data and model fit results.

The results of the analyses are automatically stored in the database. The reporting application then extracts this information, converts the $I_{c}$ values to $I_{c r}$ 's (the critical current at the specified reference temperature, $T_{\text {rof }}$ ), and computes the peak field, $H$, experienced by the sample (the sum of the applied field and the self-field [2]). Then the program performs a linear fit to the $I_{\mathrm{ct}}(H)$ values to calculate $I_{\mathrm{ct}}$ at the reference field. See Fig. 6 .

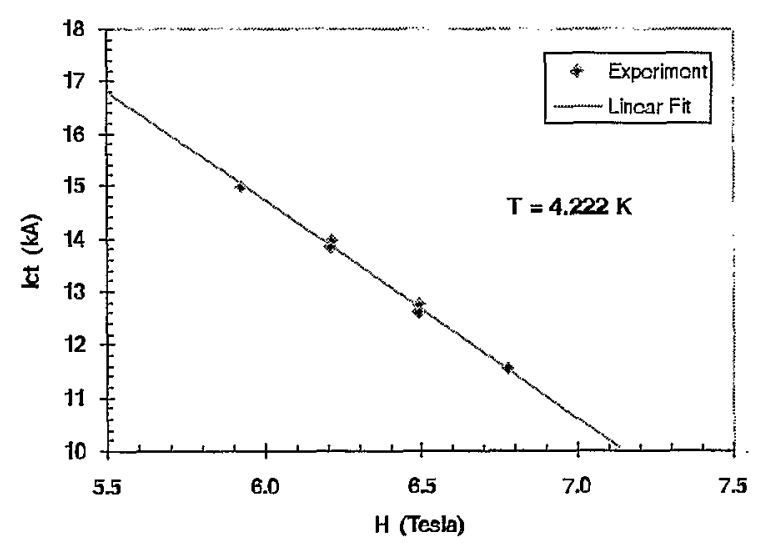

Figure 6. Linear fit to experimental $I_{\mathrm{ct}}$ results.

Other programs access the database to create plaintext files of results and reports and then transfer these to
CERN. A web-based status page is under development which will always be current, since it uses Active Server Page technology to obtain information directly from the database whenever the page is accessed.

\section{SUMMARY AND CONCLUSIONS}

Our experience to date indicates that a databasecentered approach to this data intensive cooperative program is both practical and feasible. The state of development of commercial operating system components for distributed applications, software development language tools, data analysis applications, and database servers is sufficiently mature that users can develop and maintain sophisticated client/server applications.

Using the database as the organizing structure around which software is developed for carrying out the many tasks of the collaborative effort unifies all the elements of the process and makes it possible for the users to have a comprehensive understanding of the state of the project.

The principal disadvantage is that this approach requires a rather complete understanding of what kinds of information are to be collected. If there has already been considerable experience with the procedures that need to be carried out and the difficulties that can possibly arise in acquiring and entering the data, then this approach is especially appropriate. If, on the other hand, there are many unknowns regarding what data it is necessary to collect and what difficulties may occur in collecting the data, a database-centered architecture may be less useful. As all the applications depend on the database tables, if it is necessary to frequently change the structures of these tables during the initial stages of the project to satisfy the requirements of one or another of the applications, these changes have a cascading effect and may require that other applications be updated.

However, this is not a fault of the database-centered approach itself, but merely an indication of the interrelatedness of the various tasks.

In general this data-driven method is unsuited for new exploratory investigations of an open-ended nature, but is well suited for organizing repetitive experimental and reporting procedures of a known complexity.

\section{ACKNOWLEDGMENTS}

The authors wish to thank A. Verweij and L. Obeli (CERN) for useful discussions.

\section{REFERENCES}

[1] R. McClusky, K. E. Robins, and W. B. Sampson, "A Nb 3 Sn High Ficld Dipole," IEEE Trans. On Magnetics, Vol. 27, p. 1993 (1991).

[2] M. Garber, et. al., "The Effect of Self-Field on the Critical Current Determination of Multifilamentary Superconductors," IEEE Trans.on Magnetics, Vol. 25, p. 1940 (1989). 